\title{
Análisis ontosemiótico \\ de un problema que \\ promueve la puesta \\ en funcionamiento del \\ razonamiento conjetural
}

Sonia Gallo, Silvia Catalina Etchegaray

y María Elena Markiewicz

\begin{abstract}
Resumen
Este trabajo se enmarca en una investigación en Didáctica de la Matemática, cuyo objetivo general es el análisis de los procesos argumentativos para elaborar y contrastar conjeturas en la clase de matemática de 4to. año de la escuela secundaria. Para llevar a cabo nuestro objetivo seleccionamos una serie de situaciones-problemas que promueven el funcionamiento del razonamiento conjetural en los alumnos y realizamos un análisis a priori de las mismas que se constituyó en un significado de referencia pretendido para el análisis posterior de los significados personales de los alumnos al enfrentarse a este tipo de situaciones. Una de estas situaciones y sus respectivos análisis se presentarán en este trabajo.

Como marco teórico-didáctico para llevar a cabo esta investigación hemos tomado herramientas del Enfoque Onto-semiótico del Conocimiento y la Instrucción matemáticos (EOS), así como también algunas investigaciones que abordan cuestiones específicas y transversales acerca del razonamiento conjetural.

Los análisis realizados ponen de manifiesto la complejidad ontosemiótica de este mega proceso de producción de conjeturas, la dependencia de este proceso respecto de la diversidad de objetos y de procesos cognitivos involucrados, así como también los conflictos semióticos potenciales y efectivos, todo lo cual brinda herramientas para reflexionar sobre algunas pautas para mejorar el grado de idoneidad epistémica, cognitiva e instruccional de la situación planteada y analizada.
\end{abstract}

Palabras clave: argumentaciones, razonamiento, conjeturas, análisis ontosemiótico. 


\section{Abstract}

This work is part of a research in Mathematics Didactics, whose general objective is the analysis of argumentative processes to elaborate and contrast conjectures in the mathematics class of the 4th year of high school. To carry out our objective we select a series of situations-problems that promote the functioning of the conjectural reasoning in the students, and we perform an a priori analysis of them that will constitute a meaning of intended reference for the later analysis of the personal meanings of the students when facing this type of situations. One of these situations and their respective analyzes will be presented in this work.

As a didactic theoretical framework to carry out these analyzes, we have taken tools from the Ontosemiotic Approach to Mathematical Knowledge and Instruction (OSA), as well as from research that addresses specific and transversal questions about conjectural reasoning.

The analyzes carried out reveal the ontosemiotic complexity of this mega process of production of conjectures, the dependence of this process on the diversity of objects and cognitive processes involved as well as the potential and effective semiotic conflicts, all of which provide tools for reflect on some guidelines to improve the degree of epistemic, cognitive and instructional suitability of the situation posed and analyzed.

Keywords: argumentation, reasoning, conjecture, ontosemiotic analysis.

Autores: Gallo, Sonia ${ }^{(1)}$ - Etchegaray, Silvia Catalina ${ }^{(2)}$ - Markiewicz, María Elena ${ }^{(2)}$

${ }^{(1)}$ Dirección: Blas Parera 7590. Santa Fe. Argentina

Lugar de trabajo: EETP № 633. «Centenario de Bolivia». Santa Fe

Teléfono de contacto: 342 548-7384

Dirección electrónica: soniagallo_@hotmail.com

(2) Dirección: Ruta Nacional 36 Km 601. Río Cuarto. Córdoba. Argentina

Lugar de trabajo: Universidad Nacional de Río Cuarto

Teléfono de contacto: 0358-4676228.

Dirección electrónica: setchegaray@exa.unrc.edu.ar; mmarkiewicz@exa.unrc.edu.ar 


\section{Introducción}

La problemática sobre la argumentación en matemática ha suscitado gran interés en la investigación en educación matemática. En las últimas décadas se han realizado diversos estudios (Balacheff, 2002; Boero, 1999; Recio y Godino, 2001; Mariotti, 2006; Pedemonte, 2007; Boero, Douek, Morselli y Pedemonte, 2010; Arzarello, Bartolini, Bussi, Leung, Mariotti y Stevenson, 2012) que abordan esta problemática desde distintas perspectivas, muchos de ellos poniendo el énfasis en las relaciones entre la argumentación y la prueba y otros que hacen foco en las argumentaciones que plantean los estudiantes de distintos niveles educativos. Asimismo, estudios locales (Panizza, 2005; Duarte, 2010: Barreiro, Carnelli, Falsetti y Leonián, 2012, Markiewicz, 2007, Markiewicz y Etchegaray, 2012) han abordado también esta problemática, poniendo de manifiesto la necesidad de promover, en las clases de matemática de la escuela secundaria o en estudios preuniversitarios, espacios que permitan desarrollar la argumentación y los modos de razonamientos deductivos y no deductivos.

En este sentido, y en el contexto de la escuela secundaria argentina, Markiewicz (2007) plantea como problema didáctico la escasez de espacios en la clase que promuevan el razonamiento conjetural -entendido como aquel que permite elaborar, contrastar y reformular conjeturas (Polya, 1954) - las generalmente pocas situaciones y oportunidades que se le dan al alumno para intuir, conjeturar y validar las afirmaciones formuladas y, sobre todo, para reflexionar sobre lo realizado. En la presente investigación ampliamos y precisamos esta problemática, señalando que, en general, en el ámbito de la clase no se analizan los procesos argumentativos que los alumnos podrían utilizar (y los que efectivamente utilizan) en la resolución de este tipo de situaciones.

Todo esto conlleva a que, tal como lo indica Gascón (2002), estas fases exploratorias de la actividad matemática queden muy debilitadas y casi exclusivamente a cargo del alumno; reduciéndose, además, la posibilidad del docente para anticipar y ayudar a superar posibles dificultades que puedan surgir en los procesos argumentativos que utilizan los alumnos, con el fin de movilizar la construcción del conocimiento matemático en el aula.

En este sentido, el objetivo general del estudio en el que se enmarca el presente artículo, es el análisis de los procesos argumentativos para elaborar y contrastar conjeturas en una clase de matemática de 4to.año de la escuela secundaria.

Para el logro del objetivo planteado realizamos, primero, una selección y análisis de situacionesproblemas que promueven la puesta en funcionamiento del razonamiento conjetural en los alumnos. Este análisis nos permitió contar con un significado de referencia pretendido para luego analizar los significados personales de los alumnos al enfrentarse a este tipo de problemas.

Como marco teórico-didáctico para realizar estos análisis (tanto a priori de la situación como a posteriori de los protocolos de respuestas de los alumnos) hemos tomado el Enfoque Ontosemiótico del Conocimiento y la Instrucción matemáticos (EOS), el cual nos brinda herramientas teóricas para realizar un minucioso análisis de las prácticas (institucionales y personales) ligadas a los problemas seleccionados que permite explicitar tanto la compleja trama de relaciones que se ponen enjuego al elaborar conjeturas como los procesos duales que cada momento del trabajo conlleva.

Si bien, como anticipamos, el objetivo fundamental de nuestra investigación está ligado al estudio de los procesos argumentativos de los alumnos al elaborar conjeturas, recordemos, como destaca Panizza (2005), que el razonamiento no puede ser estudiado independientemente de los contenidos matemáticos puestos en juego. Las formas de razonar de los alumnos, según esta autora, están ligadas tanto con los conocimientos que los alumnos ponen a funcionar, sus capacidades para trabajar con diferentes representaciones de los objetos matemáticos, como con sus mecanismos de control y las asociaciones producidas por el pensamiento en lenguaje natural. Además, su forma de legitimar 
sobre la validez se encuentra ligada, entre otros factores, a las verdades aceptadas como certezas y a la disponibilidad de componentes discursivos que les permitan comunicar dichos conocimientos y argumentar sobre los mismos.

Dentro del marco de referencia teórico para analizar las situaciones seleccionadas vamos a considerar, particularmente, dos trabajos que estudian aspectos más específicos y transversales del razonamiento conjetural como los de Markiewicz (2007) y Cañadas, Deulofeu, Figueiras, Reid, y Yevdokimov (2008).

En el apartado 1 expondremos brevemente las principales herramientas teóricas que utilizamos en el análisis de las situaciones seleccionadas. En el apartado 2, presentamos una de tales situaciones junto con el análisis a priori realizado a la misma, en el apartado 3, mostraremos el análisis de los protocolos de respuestas de un grupo de alumnos y, por último, en el apartado 4, esbozaremos algunas conclusiones.

\section{Marco teórico}

Este trabajo tiene las características de una investigación esencialmente descriptiva e interpretativa. Se utiliza el método basado en el diseño o ingeniería didáctica en su sentido extendido (Godino, Rivas, Arteaga, Lasa y Wilhelmi, 2014). La metodología empleada es inherente y emergente del Enfoque Ontosemiótico del Conocimiento y la Instrucción matemáticos, EOS (Godino, 2002; Godino, Batanero y Font, 2007; Godino, 2012, Godino, 2017).

El EOS estudia los procesos de enseñanza-aprendizaje de las matemáticas desde un enfoque unificado del conocimiento matemático, abarcando diferentes dimensiones: epistémica, cognitiva, instruccional y sistémico-ecológica y adoptando las nociones de sistemas de prácticas, objeto y significado (personal e institucional) como nociones básicas de la teoría. En este sentido, el EOS define práctica matemática a «toda actuación o expresión (verbal, gráfica, etc.) realizada por alguien para resolver problemas matemáticos, comunicar a otros las soluciones, validarlas o generalizarlas a otros contextos y problemas» (Godino y Batanero, 1994: 334). Estas prácticas pueden ser idiosincráticas de una persona o compartidas en el seno de una institución. El significado de un objeto matemático es un emergente de los sistemas de prácticas realizados por una persona (significado personal) o compartidas en el seno de una institución (significado institucional) para resolver un tipo de situaciones-problemas vinculados a dicho objeto. En diversos trabajos (Godino, Font y Wilhelmi, 2008; Godino et al., 2007) se han propuesto cinco niveles de análisis aplicables a un proceso de estudio matemático (planificado o bien ya implementado), los cuales constituyen una ampliación progresiva de la capacidad de análisis del mismo. El análisis que mostraremos en este trabajo está situado en los dos primeros niveles, referidos a los objetos y procesos intervinientes en un proceso de estudio matemático:

I) El primer nivel describe los sistemas de prácticas matemáticas y objetos matemáticos primarios (previos y emergentes) que intervienen o pueden resultar de la realización de dichas prácticas: situaciones-problemas, procedimientos, definiciones, propiedades, argumentaciones, lenguaje.

II) El segundo nivel de análisis se centra fundamentalmente en ciertos procesos ${ }^{(1)}$ que intervienen (o emergen) en la realización de las prácticas. Estos procesos están íntimamente vinculados a las facetas duales en que pueden ser considerados los objetos matemáticos según el juego de lenguaje en que participan. Este nivel de análisis también focaliza en los conflictos semióticos, es decir, disparidades o desajustes entre los significados atribuidos a una misma expresión por dos sujetos (personas o instituciones) que se pueden producir en una práctica matemática (Godino, 2002).

Entre los procesos a los que hace referencia el EOS podemos mencionar:

\section{- Proceso de materialización-idealización (vin-} culado a la dualidad ostensivo-no ostensivo): un objeto ostensivo es utilizado para representar, evocar o visualizar un objeto no ostensivo ideal. 
- Proceso de particularización-generalización (dualidad extensivo-intensivo): un objeto que interviene en un juego de lenguaje como un caso particular (un ejemplo específico) y como una clase más general. Este proceso tiene relación con la dialéctica entre lo particular y lo general.

- Proceso de descomposición-reificación (dualidad sistémico-unitario): el problema global puede descomponerse en problemas elementales, donde los objetos intervinientes (unitarios) deben ser tratados como parte de un sistema. Pero, tras el proceso de estudio los conceptos y propiedades emergentes deben ser reificados, es decir, vistos como objetos unitarios a fin de ser utilizados en la resolución de nuevos problemas.

- Proceso de representación-significación (dualidad expresión-contenido): consiste en atribuir significado (contenido) a una expresión, como resultado del establecimiento de funciones semióticas ${ }^{(2)}$. Estos procesos son densos en la trama de objetos y procesos que se ponen en juego en la actividad matemática.

\section{- Proceso de personalización-institucionaliza-}

ción (dualidad personal-institucional): en una primera fase de estudio es necesario lograr que los estudiantes asuman el problema y se involucren en su resolución (personalización). Luego, mediante una adecuada gestión docente, se debe promover la institucionalización de los mismos.

Estos procesos pueden ser fuente de conflictos semióticos potenciales, que ponen al descubierto disparidad de significados y que pueden explicar las dificultades y limitaciones en los aprendizajes y las enseñanzas implementadas. En este sentido, consideramos que este constructo de la teoría aporta significativamente al análisis de la dimensión semántica del conocimiento, resultando una herramienta conceptual muy flexible.

Dado el objetivo central de nuestra investigación, también tomamos como referencias teóricas dos investigaciones realizadas específicamente sobre el razonamiento conjetural. En particular, la investigación realizada por Markiewicz (2007) donde se plantea que, si bien el razonamiento conjetural corresponde más a la esfera de lo personal, es posible identificar tipos de situaciones, procedimientos, argumentaciones, definiciones y propiedades vinculadas específicamente a este tipo de razonamiento, describiendo así, elementos praxémicos y discursivos que conforman un significado de referencia institucional sobre el razonamiento conjetural. Basándose en los trabajos de Polya (1954) y Lakatos (1978), identifica distintos tipos de situaciones que favorecen la puesta en marcha de este tipo de razonamiento, en particular aquellas en las que es necesario establecer una relación general y aquellos problemas de encontrar o de demostrar, en los cuales para su resolución es pertinente recurrir a un caso particular, un caso más general o un caso análogo. Señala, asimismo, procedimientos vinculados al razonamiento conjetural, distinguiendo aquellos involucrados en la:

\section{Elaboración de conjeturas:}

- Inducción empírica: observación de casos particulares, sistematización de los ejemplos, búsqueda de regularidades, y generalización.

- Generalización: a partir del cumplimiento de una propiedad en un conjunto de objetos, se infiere el cumplimiento de dicha propiedad en un conjunto mayor que lo contiene.

- Analogía: a partir de la similitud encontrada entre dos o más cosas en algún aspecto, se infiere la similitud de esas cosas en otros aspectos.

- Ensayo y error a partir de conjeturas ingenuas que se tienen en mente y que van siendo refutadas, una tras otra.

- Conjeturar deductivo: partiendo de una proposición emparentada, realizar una síntesis para lograr la conjetura.

\section{Contrastación de la conjetura partir de:}

- Examen de consecuencias (es decir, de una afirmación que se deduce de nuestra conjetura). Esto puede consistir en la comprobación de la validez de la conjetura en un nuevo caso particular aislado (especialización), la comprobación de la validez de la 
conjetura en un conjunto de casos, un «experimento mental contrastador» (que descomponen la conjetura primitiva en subconjeturas que abren nuevas instancias de contrastación, se parte de la conjetura primitiva y se van sacando consecuencias de ella. $\mathrm{Si}$, finalmente, se llega a una consecuencia verdadera, esto hará a nuestra conjetura más creíble).

- Examen de un posible motivo (es decir, de una afirmación de la que se desprenda nuestra conjetura primitiva).

- Examen de una conjetura rival incompatible (es decir, de una proposición que no pueda ser verdadera simultáneamente con nuestra conjetura primitiva)

- Examen de una conjetura análoga.

3. Reformulación de la conjetura a partir del hallazgo de contraejemplos (globales o locales)(3) que impliquen redefiniciones de los términos que en ella intervienen, reinterpretación del contraejemplo, restricción del dominio de validez de la conjetura, identificación de la subconjetura que es refutada por el contraejemplo y su incorporación a la conjetura como condición.

También retoma algunas definiciones vinculadas al razonamiento conjetural, tales como la de conjetura, analogía, generalización, particularización, contraejemplo, etc. y algunas argumentaciones, en tanto patrones que rigen este tipo de razonamiento, como por ejemplo el Patrón inductivo fundamental: la verificación de una consecuencia hace a una conjetura más creíble (Polya. 1954). Puntualiza asimismo propiedades características del razonamiento conjetural, describiéndolo como azaroso, provisional y controversial, marcando diferencia con el razonamiento deductivo, que es preciso, definitivo y fuera de toda discusión.

Por otra parte, el trabajo de Cañadas, Deulofeu, Figueiras, Reid y Yevdokimov (2008) también nos fue de utilidad en nuestro análisis, dado que estos autores realizan aportaciones en relación con los procesos de elaboración de conjeturas, caracterizando distintos tipos de problemas que dan lugar a distintos tipos de conjeturas. En este sentido, los autores consideran que los problemas de tipo «abiertos»(4) promueven el descubrimiento y estimulan la formulación de conjeturas, impulsando el avance del conocimiento matemático.

Asimismo identifican distintos tipos de conjeturas en función de los procedimientos puestos a funcionar en su elaboración. En efecto, estos autores caracterizan en forma sistemática cinco tipos de conjeturas: a) la inducción empírica a partir de un número finito de casos discretos, b) la inducción empírica a partir de casos dinámicos, c) la analogía, d) la abducción y e) la conjetura basada en la percepción. A su vez, identifican los pasos que conlleva el conjeturar en cada uno de los tipos de conjeturas señaladas.

\section{Situación problema y análisis ontosemiótico a priori de la situación}

En este apartado presentaremos la situación seleccionada para el análisis, la cual tiene que ver con el conocido Triángulo de Pascal y ha sido formulada de modo tal que nos permita investigar el modo de razonar de los alumnos ante preguntas que promueven la elaboración y contrastación de conjeturas. Mostraremos el análisis de posibles sistemas de prácticas que podrían desplegar alumnos de 4 to año de la escuela secundaria, a los fines de caracterizar el significado pretendido que nos servirá como referencia para el análisis de significados personales de los alumnos.

\section{Problema: Triángulo de Pascal}

Este triángulo numérico es conocido con el nombre de triángulo de Pascal y se construye siguiendo un proceso determinado. 


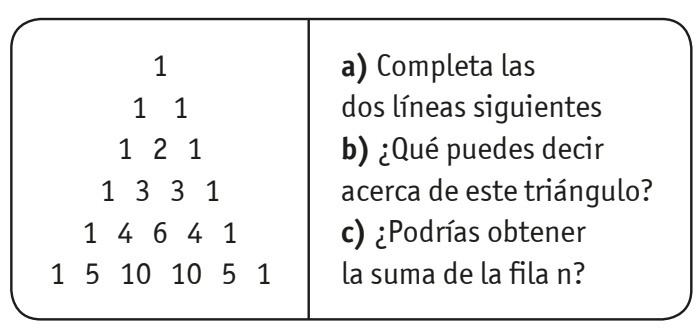

Tabla 1.

Problema.

El análisis ontosemiótico del problema presentado en la Tabla 1 nos permite estudiar y reconocer los sistemas de prácticas, objetos matemáticos y procesos intervinientes en la misma, como así también, detectar conflictos semióticos potenciales. En particular, este tipo de análisis pondrá al descubierto elementos de significado correspondientes específicamente al razonamiento conjetural (más es- pecíficamente procedimientos y argumentaciones ligados al mismo) pero permitiendo ver la íntima relación con los demás objetos y procesos puestos en funcionamiento en la tarea. Asimismo, el análisis de los potenciales conflictos semióticos pondrá de manifiesto algunos conflictos directamente vinculados a la forma de razonar al momento de elaborar conjeturas, pero que tampoco son independientes de los objetos, representaciones, lenguajes puestos en juego en la resolución de la tarea.

\subsection{Análisis de objetos primarios}

En este primernivel de análisis, como ya hemos adelantado, explicitaremos los seis tipos de entidades primarias (tareas, procedimientos, definiciones, propiedades, argumentaciones, lenguaje) como configuraciones de objetos intervinientes y emergentes de los sistemas de prácticas puestos a funcionar a partir de la situación propuesta (Tabla 2).

\begin{tabular}{|l|l|}
\hline $\begin{array}{l}\text { Situación - } \\
\text { Problema }\end{array}$ & $\begin{array}{l}\text { Se presentan una serie de números dispuestos en filas siguiendo una forma triangular, } \\
\text { explicitando que esta disposición recibe un nombre particular: «Triángulo de Pascal» y } \\
\text { que el mismo se construye siguiendo un proceso determinado. } \\
\text { Se subdivide el problema en tres subproblemas: } \\
\text { - En el primero se pide completar las siguientes dos filas del triángulo. Con esto se pretende } \\
\text { el análisis de alguna forma de construcción del triángulo a través de la exploración de } \\
\text { algunas primeras regularidades. }\end{array}$ \\
$\begin{array}{l}\text { - Elsegundo subproblema: «¿Qué puedes decir de este triángulo?» fue reformulado en forma } \\
\text { de un problema «abierto» a fin de promover la elaboración de una variedad de conjeturas } \\
\text { que apuntan a diferentes dimensiones del objeto (gráficas, aritméticas, geométricas). } \\
\text { - En el tercero, se busca obtener algún tipo de generalización que logre describir mate- } \\
\text { máticamente a qué es igual la suma de una fila cualquiera, a la que se denota como fila } n .\end{array}$ \\
\hline $\begin{array}{l}\text { Procedimientos } \\
\text { (en particular } \\
\text { indicamos con } \\
\text { RC aquellos } \\
\text { procedimientos } \\
\text { específicamen- } \\
\text { te vinculados al } \\
\text { razonamiento } \\
\text { conjetural) }\end{array}$ & $\begin{array}{l}\text { En el ítem a): } \\
\text { - Establecimiento de relaciones basadas en la percepción (RC), como por ejemplo que, los } \\
\text { «extremos» de cada una de sus «filas» están formadas por 1 (unos). } \\
\text { - Observación de uno o más casos particulares y búsqueda de regularidades (RC) para } \\
\text { elaborar un criterio de formación de los números «interiores» del triángulo, por ejemplo: } \\
\text { «puedo obtener un número del interior sumando los números que están por encima de él». } \\
\text { - Realización de los cálculos aritméticos necesarios para completar las filas del triángulo. } \\
\text { - Completar los números correspondientes a las dos últimas filas. }\end{array}$ \\
\hline
\end{tabular}




\begin{tabular}{|c|c|}
\hline & 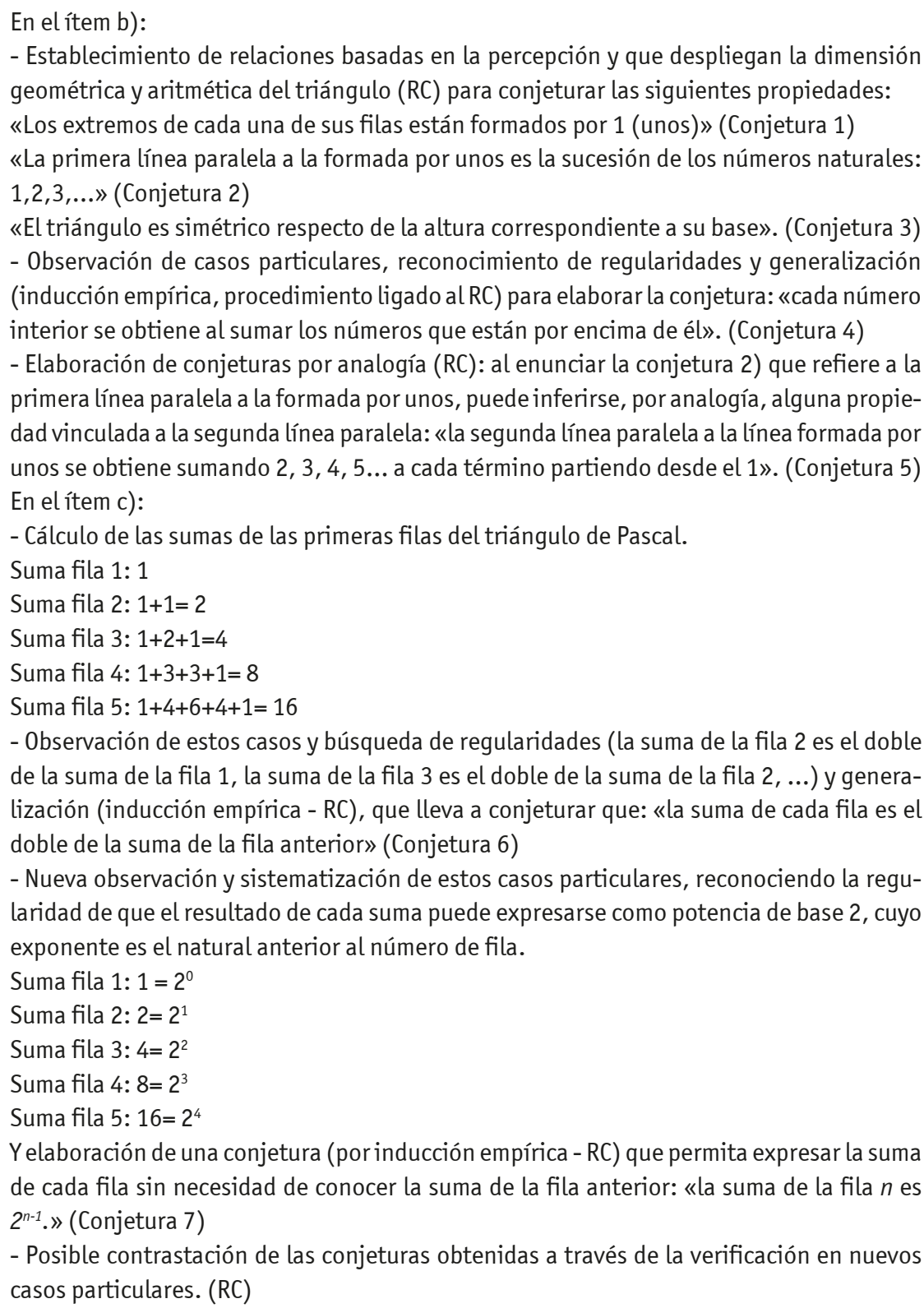 \\
\hline $\begin{array}{l}\text { Definiciones } \\
\text { /conceptos }\end{array}$ & $\begin{array}{l}\text { Previos: Sucesión de números naturales; operaciones con números naturales (adición, } \\
\text { multiplicación, potenciación); doble; triángulo y sus elementos (vértices, extremos, lados, } \\
\text { líneas, filas, altura). } \\
\text { Emergentes: Triángulo numérico de Pascal. }\end{array}$ \\
\hline Propiedades & $\begin{array}{l}\text { Previas: Simetría de un triángulo. Al razonar por analogía, la propiedad que se toma como } \\
\text { base es previa para poder conjeturar la siguiente. } \\
\text { Emergentes: Cada una de las propiedades conjeturadas: de } 1 \text { a } 7 .\end{array}$ \\
\hline $\begin{array}{l}\text { Argumenta- } \\
\text { ciones }\end{array}$ & $\begin{array}{l}\text { Las argumentaciones utilizadas corresponden a razonamientos de tipo conjetural (RC): } \\
\text { - Inferencias basadas en la percepción (en el caso de las conjeturas } 1,2 \text { y } 3 \text { ) }\end{array}$ \\
\hline
\end{tabular}




\begin{tabular}{|l|l|}
\hline Lenguaje & $\begin{array}{l}\text { - Inferencias realizadas por inducción empírica: observación, búsqueda de regularidades y } \\
\text { generalización (en el caso de las conjeturas 4,6 y 7) } \\
\text { - Inferencias por analogía (en el caso de la conjetura 5) } \\
\text { - Examen de consecuencias: se argumenta a través de la verificación de conjeturas en otros } \\
\text { casos particulares. Implícitamente se está utilizando el patrón inductivo fundamental del } \\
\text { RC (la verificación de una consecuencia de una conjetura hace a la misma más creíble) }\end{array}$ \\
\hline $\begin{array}{l}\text { Que se pone a funcionar: ordinario/coloquial; gráfico (disposición triangular propia del } \\
\text { objeto de análisis); numérico (ligado a los elementos intervinientes en el triángulo); alge- } \\
\text { braico (referido a la suma de la fila n); aritmético/simbólico (signos y símbolos propios del } \\
\text { lenguaje matemático relacionado a las operaciones aritméticas involucradas) } \\
\text { Emergente: Coloquial/específico (al expresar las propiedades que subyacen en el triángulo } \\
\text { de Pascal, y la manera de describir los objetos en b)); algebraico (relacionado a la tarea de } \\
\text { generalización propuesta en c)) }\end{array}$ \\
\hline
\end{tabular}

Tabla 2.

Entidades Primarias.

\subsection{Análisis de procesos y conflictos}

En este segundo nivel de análisis consideraremos los procesos duales y conflictos semióticos potenciales en la realización de esta situación-problema. Los mismos se particularizan en la Tabla 3.

\begin{tabular}{|c|c|}
\hline Procesos & Conflictos semióticos \\
\hline $\begin{array}{l}\text { Proceso de materialización-idealización } \\
\text { - Un ostensivo como el triángulo numérico está representando } \\
\text { un objeto ideal, que es el Triángulo de Pascal, esto es una } \\
\text { disposición triangular de números con «infinitas» filas que } \\
\text { se construye de determinada manera. } \\
\text { - Las expresiones escritas de las propiedades del triángulo, } \\
\text { apelando a términos como: «extremo», «encima de él», «líneas } \\
\text { paralelas», constituyen la materialización de las propiedades } \\
\text { conjeturadas, que subyacen como objetos ideales (no osten- } \\
\text { sivos), en la resolución de la tarea propuesta. }\end{array}$ & $\begin{array}{l}\text { - No poder materializar, en expresiones orales } \\
\text { y/o escritas, las propiedades conjeturadas; } \\
\text { evidenciar ambigüedades o imprecisiones } \\
\text { entre los términos «extremos», «filas», } \\
\text { «líneas», que dificultan la comunicación de } \\
\text { las producciones a fin de dar cuenta de las } \\
\text { exploraciones realizadas. }\end{array}$ \\
\hline $\begin{array}{l}\text { Proceso de particularización-generalización } \\
\text { - Cada número particular del interior del triángulo de Pascal } \\
\text { se obtiene a partir de un procedimiento general, descripto } \\
\text { como la suma de los números que están «por encima de él». } \\
\text { - Algunas de las regularidades y propiedades que se obtienen } \\
\text { a lo largo de la resolución, son producto de procesos de gene- } \\
\text { ralización, realizados a partir de la observación y exploración } \\
\text { de casos particulares, procedimientos a su vez asociados } \\
\text { específicamente al razonamiento conjetural. } \\
\text { - Elanálisis de la suma de cada fila del triánqulo de Pascal, obser- }\end{array}$ & $\begin{array}{l}\text { - Conflictos ligados a la búsqueda y elección de } \\
\text { casos particulares adecuados y representativos } \\
\text { que permitan llegara una generalización válida. } \\
\text { - Conflictos asociados al registro escrito } \\
\text { del lenguaje matemático/algebraico para } \\
\text { enunciar el producto de los procesos de } \\
\text { generalización, por ejemplo para la escritura } \\
\text { de la expresión algebraica } 2^{n-1} \text { como resul- } \\
\text { tado de la suma de la fila } n \text {. Lo cual asocia }\end{array}$ \\
\hline
\end{tabular}


vadas estas últimas como elementos particulares (extensivos) permite enunciar una propiedad general (intensivo) que se verifica para todas las filas del triángulo (procedimiento también vinculado al razonamiento conjetural). Si bien el significado pretendido en el ítem c) es la expresión algebraica $2^{n-1}$ como resultado de la suma de la fila $n$ del triángulo de Pascal, las generalizaciones pueden variar desde la afirmación de que todos los números hallados son pares o el hallazgo de una relación de recurrencia (expresando que cada suma se obtiene como el doble de la suma de la fila anterior), hasta el enunciado coloquial de que cada suma es una potencia de 2 o el establecimiento, además, de la relación del exponente con el número de fila.

Proceso de descomposición-reificación

- El triángulo de Pascal (nuestro objeto de estudio) es el emergente de un proceso de descomposición-reificación, donde sus elementos: números, filas, líneas, necesitan ser analizados como elementos de un sistema.

- Todo el triángulo se presenta como un sistema de números donde subyacen regularidades y propiedades. Si bien sus elementos son objetos unitarios deben ser pensados como parte de ese sistema para poder determinar cada número según su ubicación, o sea se debe someter cada objeto a la lógica del sistema. Además, si bien es cierto que el triángulo y sus elementos deben ser pensados como objetos sistémicos, luego todos esos elementos deben ser reificados para comprender el triángulo de Pascal como un objeto unitario.

- La descomposición del triángulo en filas/líneas permite ver que ciertas regularidades (como la sucesión numérica en la primera línea paralela a la línea formada por unos) sugieren el cumplimiento de propiedades análogas en otras filas/líneas, al considerar dichos elementos como parte de un sistema.

\section{Proceso de representación-significación}

- El desarrollo de la situación se apoya en la representación de un triángulo, no como objeto geométrico, sino como la de disposición gráfica de los números que evoca la representación de un triángulo. Por eso, alguno de los objetos que van a describir su proceso de formación o las propiedades podrán hacer referencia a él, tomando significados particulares y propios para este contexto, como lados, líneas, extremos.

- Las propiedades enunciadas dan cuenta del significado atribuido a las representaciones realizadas, ya sea como elementos de sucesiones, como conjuntos de números, como elementos ubicados sobre líneas.

- En el ítem c) en particular es necesario asignar un significado a los resultados de las sumas de cada fila como potencias de 2 , cuyo exponente, además es el número anterior al número de fila considerado. a este conflicto no solo con los procesos de particularización-generalización sino con el de materialización-idealización.

- No considerar necesaria la búsqueda de una expresión más general que permita hallar cualquier suma sin necesidad de conocer la suma de la fila anterior. En otras palabras, no lograr diferenciar entre el particular representado por la suma anterior a otra particular, con el particular genera que puede otorgar la suma de cualquier fila $n$.

-La consideración de los números, de las filas de las líneas como objetos unitarios, sin lograr interpretarlos en el sistema de números, puede llevar a que los estudiantes conjeturen propiedades que no se verifican en todo el triángulo o a no poder hallar regularidades ni realizar analogías entre propiedades que se cumplen en una y otra fila o línea.

- Dificultades o ambigüedades en el otorgamien to de significado (contenido) a expresiones de enunciado tales como «fila n» (entendida en este problema como una fila arbitraria de un triángulo que tiene infinitas filas), lo cual implicaría considerar a n como un arithmo y no como un parámetro; «suma de la fila n» y a algunos términos utilizados para enunciar las conjeturas (tales como «lados», «líneas», «filas»).

- No poder asignar a los resultados de las sumas de cada fila el significado de ser una potencia de 2, cuyo exponente es el número anterior a número de fila.

Tabla 3.

Procesos y Conflictos. 
Estos dos niveles de análisis epistémico del problema nos permite dar cuenta de la complejidad ontosemiótica, es decir la variedad de objetos y procesos que se deben poner en interrelación para la resolución del mismo. Con el siguiente párrafo se trata de poner de manifiesto esta interrelación para uno de las subproblemas planteados, destacando en cursiva nuestra referencia a los objetos y procesos que serían necesarios para su resolución. Para dar respuesta al problema referido a la suma de la fila $n$, como hemos visto, no sólo se deben tener disponibles las definiciones de suma y de fila $n$, sino que se debe otorgar contenido a la expresión del lenguaje: «suma de la fila $n$ » como suma de una fila cualquiera. Se debe particularizar para proceder a observar qué pasa con la suma de cada fila en particular (vista como objeto unitario), realizar los cálculos necesarios (las sumas de cada fila), para reconocer luego a todas las filas como parte de un sistema y poder hallar una regularidad en todos los casos observados. Esto requiere significar y expresar cada resultado obtenido como potencia de 2 (teniendo que tener disponible para ello la definición de potencia) para luego, generalizar este resultado: «la suma de cualquier fila es una potencia de 2». Sin embargo para hacer operativa esta generalización, sería necesario realizar una nueva particularización que permita observar la regularidad: que en cada caso el exponente del 2 es el número natural anterior al número de fila y volver a generalizar buscando una manera de materializar la propiedad conjeturada: «La suma de la fila $n$ es $2^{n-1}$ ».

\section{Análisis de sistemas de prácticas personales del problema}

El problema fue planteado en un curso de 4to. año de una escuela secundaria de la provincia de Santa Fe (Argentina). En este apartado mostraremos el análisis realizado al sistema de prácticas personales desarrollado por un grupo de tres alumnos de dicho curso con el objetivo de ejemplificar el modo de análisis que se desarrolló en esta investigación. Cabe destacar que se ha realizado este tipo de análisis a diferentes sistemas de prácticas logrados por los diferentes grupos de alumnos, que permiten observar ciertos invariantes sobre la problemática investigada.

\section{Resolución del grupo de estudiantes (E1-E2-E3)}

En este registro escrito (figura 1) aparecen indicados incisos d), e) yf), que en realidad corresponden respectivamente a (os incisos a), b) y c) del enunciado. Además del registro escrito de la producción de los estudiantes y dada la característica inherente del objeto investigado: las argumentaciones de los alumnos y los elementos de significado que sostienen dichas argumentaciones, consideramos fundamental incluir algunos diálogos que se llevaron a cabo en el grupo y que ellos mismos grabaron con su celular a pedido de la profesora, pues ayudan a entender el significado logrado.

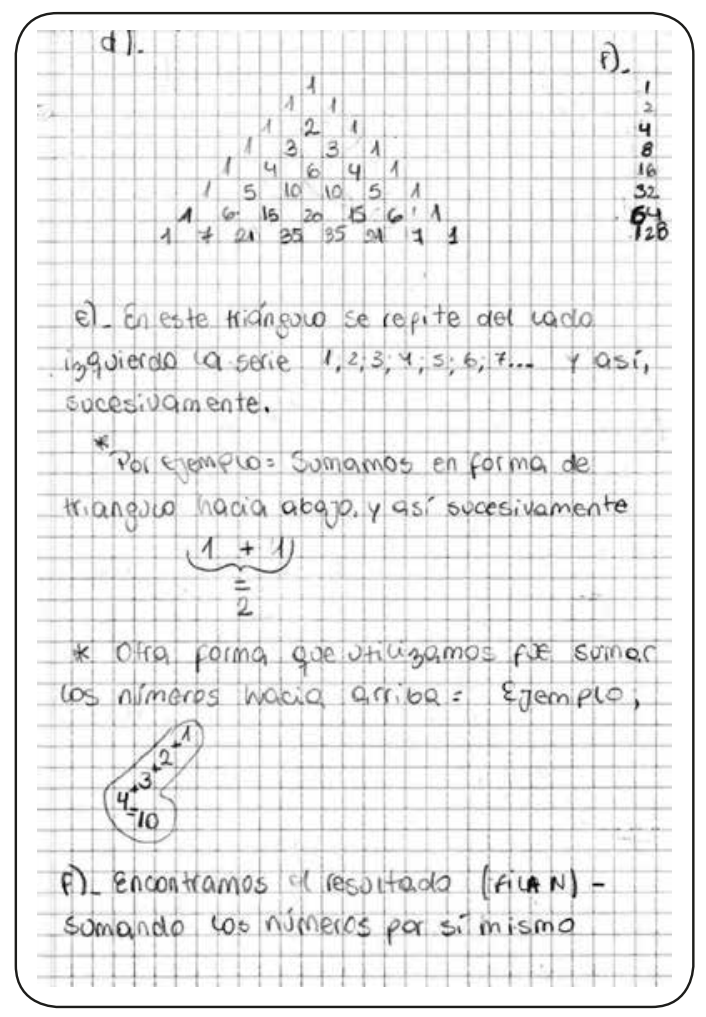

Figura 1.

Resolución de los estudiantes. 
A continuación mostraremos el primer nivel de análisis realizado al sistema de prácticas de este grupo de alumnos:

\section{Procedimientos}

En el primer ítem de la situación planteada se pide que completen las dos filas siguientes del triángulo de Pascal. Los procedimientos que utilizan para completar estas dos filas se ponen de manifiesto en el siguiente diálogo entre los alumnos y algunos de ellos quedan descriptos en la resolución del ítem siguiente.

- E1: Completemos primero los de afuera que son unos (mientras completan los extremos de las filas siguientes)

- E2: Estos unos también nos tienen que servir para completar lo de adentro... habrá que hacer algunas operaciones... fijate... También la forma de triángulo tiene que tener algo que ver...

- E3: Si sumás estos dos unos te da dos (haciendo referencia a los unos, elementos de la segunda fila, que sumados dan el dos como elemento central de la tercera fila).

- E2: Pero si sumamos 1+1+1 nos da 3... (señalando los extremos de las tres primeras filas cuya suma da el 3 ubicado como tercer elemento de la cuarta fila)

- E1: $Y$ si sumamos 1+1+1+1 nos da 4, que es el elemento de la fila que sigue...

-E2: Yasí podemos seguir con todos... y completamos los números de adentro.

(Completan los elementos de la primera línea paralela a la formada por unos, los 6 y los 7 )

- E3: Pero de esa forma no vamos a llenar todo el triángulo...., también tendríamos que ver que pasa hacia abajo... porque pide las filas siguientes... lo que nos dieron tiene que servirnos...

- E1: Mirá acá... 2x3 me da 6 que es un número de adentro y forman un triángulo... a lo mejor todos se puedan hacer multiplicando algo en forma de triángulo....

- E2: Pero mirá acá 3+3 también me da 6 y también forman un triángulo.

- E3: Como yo les dije... con los de arriba hago los de abajo... también con 6 y 4 hacés el 10... y también sirve para la punta $3+1$ te da 4, y 4+1, 5. con los números formas un triángulo... ves?

- E2: Sí, sí!!! ya está... es así!!!!

- E1: Dale, escribamos... qué sigue entonces... después del 6....

- E2 y E3: 15 porque es $5+10 \ldots, 20$, que es $10+10 \ldots$ y otra vez 15 (completan los elementos faltantes de la séptima fila)

- E1:y la otra...1, 7, 21, 35, (completan los elementos faltantes en la octava fila)

Estos diálogos muestran que los estudiantes:

- Establecen relaciones basadas en la percepción ( «los de afuera son unos»)

- Observan casos particulares buscando establecer regularidades, por ejemplo:

Que si se suman los dos «unos» de la segunda fila da como resultado el 2 que es el elemento del centro de la tercera fila, que si se suman los «unos» de los extremos de las 3 primeras filas $1+1+1$ da 3 (el tercer elemento de la cuarta fila) y que si se suman los «unos» de los extremos de las cuatro primeras filas da como resultado 4, que es el cuarto elemento de la fila siguiente. Es decir, hallan una regularidad, que de alguna manera intentan generalizar con la frase: «y así podemos seguir con todos...» y de este modo completan los elementos de la primera línea paralela a la formada por «unos», es decir, el 6 y el 7. Este último procedimiento no queda registrado en el escrito de los alumnos, quizás por lo que los mismos alumnos expresan en el sentido de que este procedimiento no les sirve para «llenar» todo el triángulo.

Observan también que si multiplican el 2 de la tercer fila por el 3 de la cuarta fila da el 6 de la quinta fila, planteando una posible regularidad: que «todos se puedan hacer multiplicando a lgo en forma de triángulo», aunque luego esa conjetura no se retome (quizás porque se hayan percatado de que no vale en general observando otros casos particulares). Retomando la primera observación realizada $(1+1=2)$, observan que también $3+3$ da 6 (y también «forman» un triángulo), estableciendo una nueva regularidad: «con los de arriba hago los de abajo», 
la cual verifican para otros casos particulares: «con 6 y 4 hacés el 10», y los de la «punta»: $3+1=4$, etc., y así completan los restantes elementos interiores del triángulo. Esta última regularidad es la que queda en el registro escrito del inciso $b$ ):

«Por ejemplo: sumamos en forma de triángulo hacia abajo y así sucesivamente» (ver Figura 2)

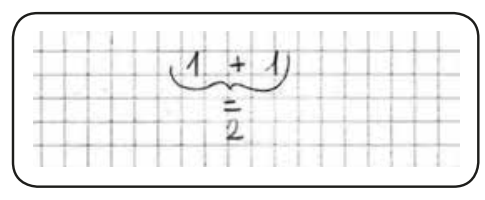

Figura 2.

La expresión «así sucesivamente» da cuenta de que, a pesar de que los alumnos han dejado escrita en su producción un solo ejemplo, han observado varios casos particulares, han hallado una regularidad y han realizado una generalización implícita, que revela la utilización de un procedimiento de inducción empírica.

El siguiente diálogo da cuenta de otros procedimientos y procesos de comunicación puesto a funcionar en este grupo al abordar el inciso b):

- E3: Profe, profe, vení...

- Docente: Y? cómo van?... ya completaron las filas... iiimuy bien!!!

-E1: Estamos acá con una duda, estamos discutiendo cómo decir esto...

- E2: Encontramos que el triángulo también se puede formar sumando «hacia arriba» (y señalan la hoja de trabajo)

- Docente: ¿Cómo?... no entiendo... explíquenme...

- E2: Fijate acá, este 10 sería la suma de estos números que están arriba (y encierran con una línea los números 10, 6, 3 y 1)

- E1: Y este 10 de al lado es la suma de estos otros números (encierran con otra línea los números 4, 3, 2y1) - E2: Y probamos con todos y siempre te da... no importa la cantidad de números que pongas en la cadena de números que elijas... ponele el 35 es $20+10+4+1$ - E1:Ytambién te da si sumás para el otro lado... ese
35 también puede ser la suma de $15+10+6+3+1 \ldots$

- Docente: iiiQué bueno!!! iiigenial!!!... iiiesa es una propiedad del triángulo!!!... ahora habría que decirlo de alguna forma....

- E3: Pero no sabemos cómo explicar...

- E1: ¿Podemos hacer un ejemplo?... porque no vamos a escribir todos los que encontramos...

- E2: Pero además tenemos que ver porque no sé si se va a entender si ponemos ejemplos...

- Docente: Bueno... ¿se animan a escribir algo de todo esto que descubrieron?... intenten...

(Mientras la profesora recorre otros grupos, los alumnos realizan su producción escrita y vuelven a solicitar la intervención docente)

- E1: Mirá, escribimos: «otra forma que utilizamos fue sumar los números hacia arriba»

- E3: Así no se entiende...hay que poner un ejemplo!!! Y dejar los números como están dibujados con la forma que tienen en el triángulo...

- Docente: Dale!!! Genial!!! Cómo quedaría??

(Una de los estudiantes escribe el ejemplo que da cuenta la producción escrita, conservando la disposición que tenía originalmente y agregando signos aritméticos «+» $\mathrm{e}$ «=»)

- E3: Ahora si se entiende!!!

- E2: Profe, ¿te parece que se entiende? la vamos a dejarasí...

Como se desprende de este diálogo, los alumnos observan casos particulares: 10 es la suma de los números que están arriba $(6+3+1), 10=4+3+2+1$, hallando otra regularidad que generalizan ( $«$ probamos con todos y siempre te da... no importa la cantidad de números que pongas en la cadena de números que elijas...») y contrastan en otros casos particulares, por ejemplo 35 es 20+10+4+1. Esto queda escrito en la resolución como: «Otra forma que utilizamos fue sumar los números hacia arriba. Ejemplo: ...»

También en este inciso b), a través de la percepción, conjeturan que «En este triángulo se repite del lado izquierdo la serie 1,2,3,4,5,6 y así sucesivamente», quedando esto registrado en su producción escrita. En ítem c), los procedimientos utilizados por los alumnos fueron: 
- Sumar los números de cada una de las 8 primeras filas del triángulo.

- Sistematizar estos resultados en una columna en el costado superior de la hoja.

- Observar una regularidad y generalizarla, explicitándola de la siguiente manera: «Encontramos el resultado (fila N) sumando los números por sí mismos». La expresión «sumando los números por sí mismos» hace referencia al procedimiento que podría emplearse para seguir avanzando en la construcción de las sumas de las filas, como así también la relación de recurrencia encontrada en la exploración de los resultados obtenidos. Advierten que cada resultado puede obtenerse al sumar al último resultado encontrado ese mismo número, (por ejemplo, $4+4=8 ; 8+8=16 \ldots$...).

\section{Definiciones}

Las definiciones utilizadas por este grupo de alumnos son las de:

Operaciones con números naturales (adición, multiplicación); triángulo y algunos de sus elementos (lados, filas), serie (como sucesión de números)

\section{Propiedades}

Como ya hemos mencionado, hay propiedades que este grupo de alumnos perciben en el triángulo y otras que construyen a partir de la observación de casos particulares, búsqueda de regularidades y generalización (inducción empírica).

Los alumnos enuncian esas propiedades de la siguiente manera: (extraído del diálogo entre los estudiantes)

- «En este triángulo se repite del lado izquierdo la serie 1,2,3,4,5,6,7...y así sucesivamente»

- "Los de afuera son unos», haciendo referencia a que los números de los extremos de cada fila son unos.

- «Sumamos en forma de triángulo hacia abajo y así sucesivamente» (figura 2)

Observemos que en este caso la conjetura que está implícita es que «si sumamos dos números contiguos de una fila (en forma de triángulo hacia abajo) obtenemos el número que está ubicado debajo de ellos en la fila siguiente del triángulo».
- «Otra forma que utilizamos fue sumar los números hacia arriba. Ejemplo: ...».

Aquí también hay una conjetura implícita no contemplada en el significado de referencia institucional: «Cada número del interior del triángulo se obtiene sumando los números que están en la línea paralela a los unos comenzando por alguno de los que están justo por encima de él».

Y hay también algunas propiedades que están presentes en los diálogos que fueron puente de alguna manera para poder enunciar algunas de las propiedades anteriores o que fueron descartadas por no ser verdaderas, por ejemplo:

- «Los unos de los extremos y la forma de triángulo pueden servir para completar lo de «adentro» (haciendo ciertas operaciones).

- «A lo mejor, todos se pueden hacer multiplicando algo en forma de triángulo»

- «Con los de arriba hago los de abajo»

\section{Argumentaciones}

- Argumentaciones basadas en la percepción.

- Inducción empírica a partir de la observación de casos particulares, búsqueda de regularidades y generalización.

- Contrastación de conjeturas mediante la verificación en nuevos casos particulares y utilización implícita del patrón inductivo fundamental: «La verificación de consecuencias hace a una conjetura más creíble». En este sentido creemos importante destacar que no siempre los ejemplos que se explicitan a continuación de las conjeturas halladas, se proveen con el fin de contrastar la conjetura sino que los mismos se mencionan como una forma de explicar la misma. (Tal es el caso del ejemplo 10= $4+3+2+1$ explicitado a continuación de la expresión: «sumamos los números hacia arriba...»)

\section{Lenguaje}

La resolución de los alumnos se caracteriza por el predominio de los lenguajes coloquial, gráfico, numérico y aritmético.

Se puede observar que las relaciones halladas son 
expresadas mediante expresiones coloquiales, como por ejemplo: «sumamos en forma de triángulo hacia abajo y así sucesivamente...», «otra forma que utilizamos fue sumar los números hacia arriba...», las cuales los mismos alumnos reconocen que no reflejan fielmente las relaciones halladas y por ello ven la necesidad de utilizar marcas en el gráfico y expresarlas con un ejemplo (es decir, aritméticamente). Recién cuando pueden hacer esto, pueden expresar que «ahora si se entiende...». Esto vuelve a poner en evidencia la utilización del ejemplo para explicar más que para contrastar en este caso. Se destaca asimismo el uso (casi abusivo) de las expresiones coloquiales «adentro» y «afuera», lo que de alguna manera obstaculiza la correcta enunciación de algunas de las relaciones halladas. Este lenguaje no fue anticipado en nuestro significado de referencia pretendido.

El lenguaje numérico y el aritmético dan cuenta de las operaciones realizadas para completar los elementos de las filas y de la escritura de los números respetando la estructura triangular de la disposición gráfica previamente dada, de lo que se infiere que dicha forma fue utilizada en la fase de construcción. También utilizan un lenguaje simbólico-aritmético (signos y símbolos propios del lenguaje matemático relacionado a las operaciones aritméticas involucradas) tanto para la explicitación de los procedimientos como para las propiedades del triángulo, no habiendo indicios de lenguaje simbólico algebraico.

Profundizando en un segundo nivel de análisis, se pone en evidencia que estos estudiantes han transitado por diferentes procesos:

\section{- Procesos de materialización-idealización:}

Los números escritos y su disposición triangular (ostensivos) en el ítem a) refieren a la materialización de los procedimientos que se llevaron a cabo a la hora de resolver el problema, como la observación, la exploración y la búsqueda de regularidades. Las expresiones escritas, utilizando expresiones tales como: «se repite del lado izquierdo la serie 1, 2, 3, 4, 5, 6, 7 y asi sucesivamente», "sumamos en forma de triángulo hacia abajo», "sumar los números hacia arriba», «encontramos el resultado (filan) sumando los números por símismos» constituyen la materialización de ciertas propiedades que subyacen como objetos ideales (no ostensivos).

En este sentido, podemos observar, tal como lo habíamos anticipado en el análisis del significado de referencia pretendido, que hay ciertas dificultades e imprecisiones para materializar, en expresiones orales y/o escritas, las propiedades conjeturadas, por ejemplo: «En este triángulo se repite del lado izquierdo la serie 1,2,3,4,5,6,7... y asísucesivamente», utilizando las expresiones «se repite del lado izquierdo» que es ciertamente confusa, «serie» en lugar de sucesión. Es decir, hay conflictos semióticos asociados al uso de los componentes discursivos para la comunicación de la regularidad hallada.

Pero, además, y esto nos parece de suma importancia, observamos que en algunas oportunidades las propiedades halladas quedan expresadas en términos de procedimientos. Por ejemplo, decir: «sumamos en forma de triángulo hacia abajo y así sucesivamente», sin poder expresar esta regularidad en forma de una propiedad general: «si se suman dos números contiguos de una fila (en forma de triángulo hacia abajo) se obtiene el número que está ubicado debajo de ellos en la fila siguiente del triángulo ideal». Observemos que en un momento la docente les dice: «iii Esa es una propiedad del triángulo!!!... ahora habría que decirlo de alguna forma...», es decir les solicita expresamente decir en términos de propiedad. A lo que los alumnos expresan que no saben cómo explicary materializan la propiedad subyacente en términos de procedimiento.

En el caso del inciso c), este conflicto se hace más patente. Los estudiantes expresan la conjetura en términos de procedimientos y con algunas imprecisiones: «Encontramos el resultado (fila N) sumando los números por sí mismos».

\section{- Procesos de particularización-generalización}

- Tanto en el registro escrito como en los diálogos entre los alumnos y la docente se pone de manifiesto cómo ha funcionado la dialéctica entre 
lo particular y lo general. Tanto para pensar la construcción de las filas siguientes como en la enunciación de propiedades del triángulo, se analizan casos particulares para lograr algún tipo de generalización. Por ejemplo, cada número particular del interior del triángulo se obtiene a partir de un procedimiento general, descripto como «sumamos en forma de triángulo hacia abajo». Pero también esa generalización es verificada en nuevos casos particulares e incluso se ve la necesidad de ejemplificar las relaciones conjeturadas mediante nuevos casos particulares.

- En el caso del ítem c) el análisis de la suma de cada fila, observadas como elementos particulares, permite enunciar un regularidad, que intentan generalizar en la expresión «Encontramos la suma el resultado (fila N) sumando los números por sí mismos».

También aquí observamos algunos conflictos semióticos asociados a este proceso de particularización-generalización, algunos de los cuales ya habíamos anticipado como conflictos potenciales: - Conflictos ligados a la elección de casos particulares adecuados que permitan llegar a una generalización válida, como por ejemplo la consideración de la tercer línea paralela a los «unos» y la observación de cada número particular para obtener una ley general de formación de dicha sucesión.

- No lograr diferenciar entre el particular representado por la suma anterior a otra particular, con el particular general que puede otorgar la suma de cualquier fila $n$.

-También se perciben dificultades asociadas al registro escrito del lenguaje utilizado para enunciar el producto de los procesos de generalización, y la consiguiente necesidad de hacer referencia a un ejemplo (caso particular) para que se entienda la generalización a la que arribaron. Esto se ve claramente en la forma de expresar la relación general hallada: «sumamos en forma de triángulo hacia abajo y así sucesivamente» (seguida del ejemplo) u «otra forma que utilizamos fue sumar los números hacia arriba. Ejemplo:...) las cuales, como ya hemos mencionado, no pueden expresar como una propiedad general. Este conflicto, está relacionado no sólo con los procesos de particularización- generalización sino con el de materialización-idealización.

\section{- Proceso de descomposición-reificación}

Esta práctica matemática da cuenta del proceso de descomposición-reificación llevado a cabo por este grupo, que si bien va considerando los números involucrados como objetos unitarios con los que realiza cálculos según su ubicación, son a la vez, sometidos a la lógica del sistema, observando a todo el triángulo como un objeto sistémico. Siendo así el triángulo de Pascal el emergente de un proceso de descomposición-reificación de elementos unitarios (números, filas, triángulos más pequeños) que necesitan ser analizados como elementos de un sistema y luego nuevamente transformado en un objeto unitario.

Entre los conflictos relacionados a este proceso podemos destacar que en este grupo de alumnos la consideración de las «líneas» como objetos unitarios (sin verlos en el sistema de números), lleva a que no puedan realizar analogías entre propiedades que se cumplen en otras líneas, por ejemplo, el mirar como objeto unitario a la primer línea paralela a los unos, a pesar de poder percibir que se trata de la sucesión $1,2,3,4,5, \ldots$, no les permite ver que la segunda línea paralela a los unos también es una sucesión con determinadas características.

\section{- Proceso de representación-significación}

Este proceso se pone en juego en la producción y comunicación de esta resolución. Desde un principio los alumnos tuvieron que dar un significado a algunas expresiones del enunciado de la tarea, en particular a la expresión: «fila $n$ ». En la resolución y los diálogos, algunos objetos, como los números que forman las filas o las expresiones coloquiales, evocan la representación geométrica triangular referenciándola en el procedimiento utilizado para su formación, tomando elementos de la geometría («sumamos en forma de triángulo») o de las relaciones espaciales («hacia abajo»/ «sumar los números hacia arriba»/ «del lado izquierdo»), para describirlos. 
Los conflictos semióticos ligados a este proceso tienen que ver, tal como lo habíamos anticipado, con dificultades 0 ambigüedades en el otorgamiento de significación a algunos términos utilizados en el enunciado de la tarea y en su resolución. En particular la asignación de un contenido a la expresión «fila $n$ » se presentó como un conflicto semiótico efectivo en el desarrollo de esta tarea, que hizo necesaria la intervención del docente para hacer progresar la actividad matemática requerida. Asimismo, se vislumbraron conflictos semióticos vinculados a ambigüedades en el significado otorgado a los términos y expresiones utilizados para enunciar las conjeturas (tales como «serie», «lado izquierdo», «sumar en forma de triángulo hacia abajo», «sumar los números hacia arriba», «sumar los números por sí mismos»).

Debemos destacar que la atribución de significado a la expresión algebraica del enunciado requiere de cierta familiaridad con las prácticas del lenguaje específico-matemático, que aparentemente no estaban disponibles por los estudiantes al momento de enfrentarse a la tarea, y se manifestó en dificultades/obstáculos para resolverla. En este sentido el EOS pone en evidencia que las transformaciones de lenguajes no se plantean por simples traducciones, por lo que lograr el enunciado en lenguaje algebraico es mucho más que una traducción de lenguajes, sino que necesita de una transformación de significados que pone a funcionar nuevas funciones semióticas, más allá de tener una disponibilidad personal de signos o expresiones algebraicas, cuestión que en este caso, necesitó de la gestión del docente.

\section{Conclusiones}

A fin de analizar los procesos argumentativos de alumnos de 4to. año de la escuela secundaria, hemos seleccionado y reformulado una situación que consideramos potencialmente «rica» ${ }^{(5)}$ para poner en funcionamiento este tipo de procesos, en particular los involucrados en la elaboración y contrastación de conjeturas.
Situados en una dimensión epistémica, hemos realizado un análisis a priori de la situación, el cual muestra una posible red de relaciones entre los diferentes objetos, procesos duales y razonamiento empleados en una resolución pretendida para alumnos de 4to año de la escuela secundaria, el cual pone en evidencia la complejidad ontosemiótica que subyace a este mega proceso que es la argumentación, y en particular, la elaboración de conjeturas.

En este sentido, y profundizando lo expresado por Panizza (2005) respecto de que los razonamientos están ligados a los conocimientos que se ponen en juego, nuestra investigación muestra que el proceso de elaboración de conjeturas no sólo está condicionado por todos los objetos primarios que se ponen a funcionar: desde el problema, los procedimientos, las definiciones, las propiedades, el lenguajey el tipo de argumentación utilizados, sino también por los procesos duales que se deben transitar: además del proceso de particularización-generalización, los procesos de materialización-idealización, de representación-significación y de descomposición-reificación.

El análisis a priori del problema nos fue de utilidad como significado de referencia para luego analizar los significados personales de los alumnos (dimensión cognitiva), que se manifiestan en un sistema de prácticas realizadas por un grupo de alumnos ante tal situación.

Este análisis de significados personales pone en evidencia ciertas disparidades entre lo que el profesor espera (significado institucional pretendido) y lo que realmente logran hacer los alumnos (significado personal logrado), que se explican en términos de conflictos semióticos.

En relación a esta cuestión, observamos que algunos de los conflictos semióticos potenciales que se habían anticipado se hicieron efectivos en la clase (y en particular en este grupo de alumnos) como lo son, por ejemplo, aquellos ligados a la falta de elementos discursivos para poder materializar las conjeturas halladas, a la dificultad en el otorgamiento de significado a ciertas expresiones tales como «suma de la fila $n$ » 0 en la falta de una 
mirada sistémica de ciertos objetos que impiden la emergencia de otras conjeturas. Pero hay conflictos que no fueron anticipados y que nos resultan importantes de destacar y de señalar como nuevos focos de indagación: observamos la presencia, en los alumnos, de una cierta «confusión ontológica», en el sentido de que algunos objetos primarios que se pretendía que emergieran como propiedades generales (del triángulo de Pascal en este caso) terminan expresándose como procedimientos que pueden utilizarse para la formación del triángulo. Esto muestra claramente que no se logra la despersonalización ni la descontextualización esperada en la formulación de los alumnos a partir sólo de lo que el problema plantea. La persistencia de esta confusión ontológica en otras situaciones nos ayudaría a explicar, en parte, la falta de necesidad por parte de los estudiantes de validar lo que se dice ya que si la conjetura se escribe como el procedimiento realizado, no habría nada que validar. Por eso insistimos en que es necesario trabajar en ese «cambio» en la forma de enunciar la conjetura, transformando el elemento de significado de procedimiento a propiedad.

También evidenciamos una importante confusión argumentativa respecto al uso del ejemplo. En forma reiterada ese uso significa explicación y no contrastación como se plantea en nuestro marco de referencia.
Cabe destacar que estos conflictos son invariantes, no solo en las resoluciones de este problema logradas por todos los grupos de alumnos, sino también en otro tipo de problemas analizados en esta investigación.

En este sentido, creemos que es necesario un trabajo en el aula dirigido a significar una propiedad matemática como una proposición (oración, enunciado) que afirma algo general, y a reflexionar sobre el uso del ejemplo, lo cual exigiría una etapa de planificación y diseño especial.

En general, los conflictos semióticos potenciales y efectivos nos ayudan a explicar, utilizando herramientas semióticas, las diversas dificultades por las que pueden atravesar ( $y$ de hecho atraviesan) los alumnos en sus procesos argumentativos, y en particular en el proceso de elaboración de conjeturas. También nos permiten repensar las intervenciones docentes en el aula, dado que las mismas deben estar orientadas a tratar de destrabar estos conflictos semióticos para ayudar a avanzar a los alumnos en la elaboración de las conjeturas pretendidas.

Por último, creemos que el estudio realizado aporta a la problemática planteada en la introducción de este trabajo, en tanto que favorece, desde las dimensiones epistémica, cognitiva y semiótica, a la comprensión y desnaturalización de los procesos argumentativos de los alumnos en situaciones de elaboración y contrastación de conjeturas.

\footnotetext{
Notas

(1) Se entiende como proceso a una secuencia de acciones que es activada o desarrollada durante un cierto tiempo para conseguir un objetivo. En este sentido, debemos destacar que el EOS reconoce la existencia de una gran diversidad de procesos que se ponen en juego en la actividad matemática. De hecho, la emergencia de los objetos primarios de una configuración (lenguaje, problemas, procedimientos, definiciones, propiedades, argumentos) tiene lugar mediante los respectivos procesos matemáticos de comunicación, problematización, elaboración de procedimientos, definición, enunciación, argumentación. A su vez, destaca mega procesos en la actividad matemática como lo son la resolución de problemas y la modelización, entre otros.

(2) La noción de función semiótica se entiende como la correspondencia entre un objeto antecedente (expresión, significante) y otro consecuente (contenido, significado) establecida por un sujeto (persona o institución) según un criterio o regla de correspondencia (Godino, Batanero y Font, 2007).
} 
(3) Los contraejemplos globales son aquellos que refutan la conjetura original, mientras que los contrajemplos locales son aquellos que refutan a alguna de las posibles subconjeturas.

(4) Estos autores consideran problemas «abiertos» a aquellos donde se da alguna de estas tres situaciones: «a) dadas las propiedades iniciales, el problema consiste en encontrar las consecuencias de ellas, b) dadas las propiedades finales, el problema consiste en encontrar las propiedades iniciales de las cuales son consecuencia, c) sin dar ninguna propiedad del problema consiste en encontrar las propiedades relacionadas.

(5) El término «situación rica» es ambiguo ya que se usa con muchos sentidos diferentes. Utilizamos dicho término en concordancia con Font (2005), para hacer referencia a que la situación elegida supera elaprendizaje pasivo, gracias a la incorporación al proceso de enseñanza-aprendizaje, entre otros, de algunos de los siguientes aspectos: la actividad del alumno, el problema contextualizado, la organización para su enseñanza en grupos de trabajo y la posibilidad de que los alumnos visualicen diferentes regularidades.

\section{Referencias bibliográficas}

Arzarello, F., Bartolini-Bussi, M., Leung, A., Mariotti, M., \& Stevenson, I. (2012). Experimental Approaches to Theoretical Thinking: Artefacts and Proofs. En G. Hanna, \& M. de Villiers, Proof and Proving in Mathematics Education (págs. 97-146). New York: Springer. Disponible en: https://www. researchgate.net/publication/265502765_Experimental_approaches_to_theoretical_thinking_artefacts_and_proofs_With_a_response_by_Jonathan_M_Borwein_and_Judy-anne_Osborn

Balacheff, N. (2000) Procesos de Prueba en los alumnos de matematica. Colombia: Una empresa docente. Disponible en: https://hal.archives-ouvertes.fr/hal-00520133/document

Barreiro, P., Carnelli, G., Falsetti, M., Leonián, P. (2012) Acercamiento a la validación en Matemática de estudiantes de pre-grado en clases ordinarias Revista Electrónica Iberoamericana de Educación en Ciencias y Tecnología. Vol $3 \mathrm{~N}^{\circ} 2$ 2. Argentina. Disponible en: http://www.exactas.unca.edu.ar/riecyt/VOL\%20 3\%20NUM\%202/Archivos\%20Digitales/ RieCyT\%20V3\%20N2\%20Set\%202012\%20Doc\%20-7-.pdf

Boero, P. (1999) Argumentation and mathematical proof: A complex, productive, unavoidable relationship in mathematics and mathematics education. International newsletter on the teaching and learning of mathematical proof 07/08. Disponible en: http://wwwdidactique.imag.fr/preuve/ Newsletter/990708Theme/990708ThemeUK.html.

Boero, P., Douek, N., Morselli, F., \& Pedemonte, B. (2010). Argumentation and proof: a contribution to theoretical perspectives and their classroom implementation. En M.M. Pinto, \& T.F. Kawasaky (Ed.), Proceedings of the 34th Conference of the International Group for the Psychology of Mathematics Education. 1, págs. 179-204. Belo Horizonte, Brazil: PME.

Cañadas, M.C., Deulofeu, J., Figueiras, L., Reid, D. y Yevdokimov, 0. (2008) Perspectivas teóricas en el proceso de elaboración de conjeturas e implicaciones para la práctica: tipos y pasos. Enseñanza de las Ciencias, 26(3), 431-444. Disponible en: https://raco. cat/index.php/Ensenanza/article/view/132198 Duarte, Betina (2010) Cuestiones didácticas a propósito de la enseñanza de la fundamentación en matemática. La Funcion Exponencial, el Razonamiento Matematico y la Intervencion Docente en la Escuela Media. Tesis Doctoral. Univesidad de San Andrés. Buenos Aires. Argentina. Disponible en: https://www.udesa.edu.ar/usina-de-la-educacion/tesis-0

Font, V. (2005). Reflexión en la clase de Didáctica de las Matemáticas sobre una «situación rica», en Badillo, E. Couso, D., Perafrán, G., Adúriz-Bravo, A. (eds) Unidades didácticas en Ciencias y Matemáticas (pp. 59-91). Magisterio: Bogotá. Disponible en: http://www.pagvf.esy.es/index_archivos/ Situaciones\%20ricas.pdf

Gascón, J. (2002) El problema de la Educación Matemática y la doble ruptura de la Dida 'ctica de las Matemáticas. Gaceta de la Real Sociedad Matemática Española , 5(3), 673-698. Disponible en: http:// gaceta.rsme.es/abrir.php?id=125 
Godino, J.D. (2002) Un enfoque ontológico y semiótico de la cognición matemática. Recherches en Didactique des Mathématiques, 22(2.3), 237-284. Disponible en: http://enfoqueontosemiotico.ugr. es/pages/configuraciones.html

(2012) Origen y aportaciones de la perspectiva ontosemiótica de investigación en Didáctica de la Matemática. En A. Estepa, A. Contreras, j. Deulofeu, M.C. Penalva, F.J. García y L. Ordoñez (Eds.), Investigación en Educación Matemática XVI (pp. 49-68).Jaén: SEIEM. Disponible en: https://www. researchgate.net/publication/282325808 Origen_y_aportaciones_de_la_perspectiva_ontosemiotica_de_investigacion_en_didactica_de_la_matematica

(2017). Construyendo un sistema modular e inclusivo de herramientas teóricas para la educación matemática. En J.M. Contreras, P. Arteaga, G.R. Cañadas, M.M. Gea, B. Giacomone y M.M. López-Martín (Eds.), Actas del Segundo Congreso International Virtual sobre el Enfoque Ontosemiótico. del Conocimiento y la Instrucción Matemáticos. Godino, J.D. (2017) Disponible en: http:// enfoqueontosemiotico.ugr.es/civeos/godino.pdf

Godino, J.D. y Batanero, C. (1994) Significado institucional y personal de los objetos matemáticos. Recherches en Didactique des Mathématiques, 14 (3), 325-355. Disponible en: https://www.ugr.es / jgodino/funciones-semioticas/03_SignificadosIP_RDM94.pdf

Godino, J.D., Batanero, C., y Font, V (2007) The ontosemiotic approach to research in mathematics education. ZDM. The International Journal on Mathematics Education, 39 (1-2), 127-135. (Versión ampliada en español en: http://www.ugr.es/local/jgodino/indice_eos.htm)

Godino,J.D., Font ,V. y Wihelmi, M.R. (2008) Análisis didáctico de procesos de estudio matemático basado en el enfoque ontosemiótico. Publicaciones, Vol. 38: 25-49. Disponible en: https://www.ugr. es/ jgodino/indice_eos.htm

Godino, J.D., Rivas, H., Arteaga, P., Lasa, A. y Wilhelmi, M.R. (2014). Ingeniería didáctica basada en el enfoque ontológico - semiótico del conocimiento y la instrucción matemáticos. Recherches en Didactique des Mathématiques, 34 (2/3), 167-200. Disponible en: https://www.ugr.es/ jgodino/ eos/JDGodino_ID-EOS_31mayo2014.pdf

Lakatos, I. (1978) Pruebas y refutaciones: la lógica del descubrimiento matemático. Madrid: Alianza Editorial.

Mariotti, M. (2006). Proof and Proving in Mathematics Education. En A. Gutiérrez, \& P. Boero, Handbook of Research on the Psychology of Mathematics Education. Past, Present and Future (págs. 173-204). Rotterdam, The Netherlands: Sense Publishers. Disponible en: http://math.unipa.it/ grim/ YESS-5/PMEbook_MariottiNew.pdf

Markiewicz, M.E. (2007) El rol del razonamiento plausible en la enseñanza de la matemática (Versión

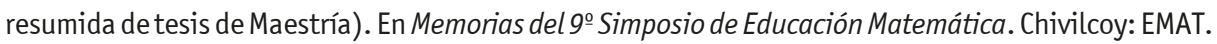

Markiewicz, M.E. y Etchegaray, S. (2012) Significados personales vinculados al razonamiento conjetural en la formación del profesor. En Ascheri, M.E., Pizarro, R.A., Ferreyra, N. y Astudillo, G. (Eds.). Memorias de la IV Reunión Pampeana de Educación Matemática. Santa Rosa de La Pampa: Ed. UNLPam. Disponible en: http://repem.exactas.unlpam. edu.ar/cdrepem12/memorias/Reportes\%20 de\%20Investigaci\%C3\%B3n/CB\%2023.pdf

Panizza, M. (2005) Razonary conocer. Aportes a la comprensión de la racionalidad matemática de los alumnos. Bs. As. Argentina: Libros del Zorzal.

Pedemonte, B. (2007). How can the relationship between argumentation and proof be analysed? Educational Studies in Mathematics, 66(1), 23-41. doi: https://doi.org/10.1007/s10649-006-9057-x Polya, G. (1954) Mathematics and Plausible Reasoning. New Jersey: Princeton University Press.

Recio, A. y Godino, J.D. (2001) Institucional and personal meanings of mathematical proof. Educational Studies in Mathematics, 48,83-99. Disponible en: http://citeseerx. ist.psu.edu/viewdoc/dow nload?doi=10.1.1.454.4579\&rep=rep1\&type=pdf 